\title{
Motion planning for high DOF anthropomorphic hands
}

\author{
J. Rosell, R. Suárez, C. Rosales, J. A. García and A. Pérez
}

\begin{abstract}
The paper deals with the problem of motion planning of anthropomorphic mechanical hands avoiding collisions. The proposed approach tries to mimic the real human hand motions, but reducing the dimension of the search space in order to obtain results as a compromise between motion optimality and planning complexity (time) by means of the concept of principal motion directions. Basically, the work includes the following phases: capturing the human hand workspace using a sensorized glove and mapping it to the mechanical hand workspace, reducing the space dimension by looking for the most relevant principal motion directions, and planning the hand movements using a sampling-based roadmap planner. The approach has been implemented for a four finger anthropomorphic mechanical hand, and some examples are included to illustrate its validity.
\end{abstract}

\section{INTRODUCTION}

Advances in robotics are producing a number of complex devices with a high number of degrees of freedom (DOF), lots of sensors, and sophisticated controllers to assure stability and a good performance. These devices include different types of robots, adapted to different environments and tasks, and among them the most representative instances are the humanoids, equipped with anthropomorphic hands with a number of DOF ranging from 12 (four fingers with 3 independent DOF each one) to 25 (five fingers with 4 independent DOF each one plus some DOF in the palm [1]). Examples of anthropomorphic hands with four fingers are the Utah/MIT Hand [2], DIST Hand [3], LMS Hand [4]; DLR Hand [5] and MA-I Hand [6], and examples with five fingers are the Belgrade/USC Hand [7], Anthrobot-2 Hand [8], NTU Hand [9], ROBONAUT [10], Shadow Hand [11], Gifu Hand [12] and Bolonia Hand 3 [13]. Good discussions about robot hands can be found in [14] and [15].

Despite the advanced features of these mechanical hands, one of the remaining problems in order to obtain a good outcome from them is the automatic determination of their movements, which are quite complex and non-evident for the human being in the space of generalized coordinates. This is a well-known motion planning problem, but in a very large dimensional space, thus some new approaches are still necessary in order to find solutions that can be really implemented and used in practice. This paper presents some developments in this line, looking for procedures that allow the automatic motion planning of anthropomorphic hands in

This work was partially supported by the CICYT projects DPI2007-63665 and DPI2008-02448.

The authors are with the Institute of Industrial and Control Engineering Technical University of Catalonia, Barcelona, Spain.jan.rosell@upc.edu

C. Rosales is also with the Institut de Robòtica i Informàtica Industrial, CSIC-UPC. A. Pérez is also with the Escuela Colombiana de Ingeniería "Julio Garavito" placed in Bogotá D.C., Colombia. a smooth way, caring for collisions with the environment as well as between the different parts of the hand.

\section{PROBLEM STATEMENT AND SOLUTION OVERVIEW}

Let $\mathcal{C}=\mathcal{C}_{\mathrm{h}}+\mathcal{C}_{\mathrm{a}}$ be the configuration space of a handarm system, where $\mathcal{C}_{\mathrm{h}}$ and $\mathcal{C}_{\mathrm{a}}$ are the configuration spaces of the hand and of the arm where it is mounted, respectively. The basic problem to be solved is the following: given the current hand-arm configuration $C_{o}$ and a final desired one $C_{d}$ (that is not necessarily a grasping configuration), find a collision free path from $C_{o}$ to $C_{d}$. The dimension of the configuration space of this problem is equal to the number of DOF of the hand plus the number of DOF of the arm, therefore conventional solutions require high computational times. In this context, the proposed approach is based on a reduction of the configuration space dimension, which is done by looking for a representative subspace $\mathcal{S} \mathcal{C}_{\mathrm{h}}$ of the hand configuration space $\mathcal{C}_{\mathrm{h}}$, and looking for continuous valid paths in the compound subspace $\mathcal{S C}=\mathcal{S C}_{\mathrm{h}}+\mathcal{C}_{\mathrm{a}}$. Of course, there may be solutions in $\mathcal{C}$ not included in $\mathcal{S C}$, thus the selection of a proper subspace $\mathcal{S C}_{\mathrm{h}}$ is a relevant step in the proposed approach. On the other hand, if a solution is found in $\mathcal{S C}$, for sure it is valid in $\mathcal{C}$.

The main consideration that supports the reduction of the problem space is that the human hand has several joint movements that are not (completely) independent, and therefore they can be associated in some way. A typical example is given by the last two joints of each finger, which (normally) cannot be moved independently; in the same way some other relations can be found analyzing the hand configuration space. This feature can be extrapolated to mechanical hands where the analysis is done by taking enough samples of $\mathcal{C}_{\mathrm{h}}$ and looking then for the direction in which the samples present the largest dispersion, which is iteratively repeated considering orthogonal directions until a new basis of $\mathcal{C}_{\mathrm{h}}$ is generated. Then, by selecting the first vectors of this basis and properly choosing a bounding box aligned with these vectors and centered in the mean value of the original set of points, a good bounded approximation $\mathcal{S C}_{\mathrm{h}}$ of $\mathcal{C}_{\mathrm{h}}$ is found.

A relevant previous work in this line [16] uses an initial set of grasping configurations to find a bidimensional grasp subspace, i.e. to characterize the configurations of the hand used to grasp different objects. This subspace is used in other works to look for grasping configurations [17], [18]. As a difference with these works, we use here an initial set of unconstrained general hand configurations in order to model all the real hand workspace and not only potential 


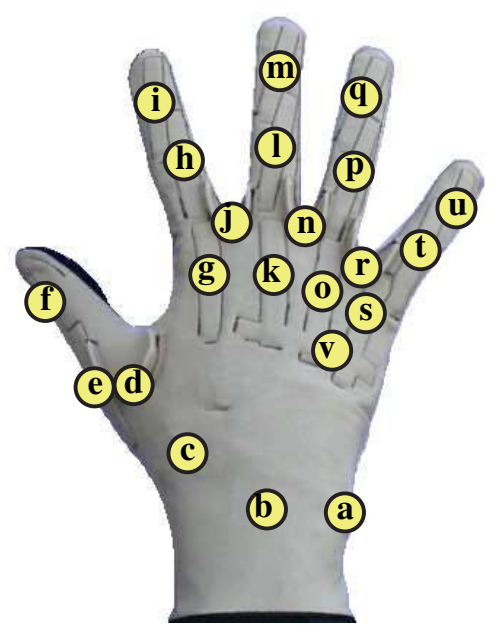

Fig. 1. Sensorized glove used to capture the operator hand workspace.

grasping configurations. The particular procedure followed to generate the set of hand configurations as well as to find a proper bounded subspace is detailed in Section III. Once the bounded subspace is determined, sampling-based motion planning techniques are used to model the free configurations and to find free paths between any two of them (Section IV). Dimensionality reduction techniques have also been used to synthesize human-like motion in graphic applications [19].

The approach followed in this work can be summarized in the following steps:

1) Use a sensorized glove to obtain samples of the human hand workspace (22 DOF) (Subsection III-A).

2) Map these samples to the configuration space $\mathcal{C}_{\mathrm{h}}$ of a mechanical hand (13 DOF) (Subsection III-B).

3) Find a representative subspace $\mathcal{S C}_{\mathrm{h}}$ of the mechanical hand configuration space $\mathcal{C}_{\mathrm{h}}$ (between 3 and $6 \mathrm{DOF}$ ) (Subsection III-C).

4) Use a sampling-based roadmap planner to model the free space of the representative subspace $\mathcal{S C}=\mathcal{S C}_{\mathrm{h}}+\mathcal{C}_{\mathrm{a}}$ (Section IV).

5) Finally, given an initial and final hand-arm configurations of $\mathcal{C}$ (not necessarily belonging to $\mathcal{S C}$ ), $C_{o}$ and $C_{d}$ respectively, connect them to the roadmap and use it to find a free path between them.

\section{MODELING HAND MOVEMENTS WITH PRINCIPAL MOTION DIRECTIONS}

\section{A. Data Aqcuisition}

The data acquisition is done using the commercial sensorized glove CyberGlove ${ }^{\complement}$ from Immersion Corporation, shown in Fig. 1. It is a fully instrumented glove that provides up to 22 high-accuracy joint-angle measurements, using resistive bendsensing technology. The 22 -sensor model has three flexion sensors per finger, four abduction sensors, a palm-arc sensor, and sensors to measure the flexion and the abduction of the wrist.

After a calibration procedure for each user's hand, the movements captured with the glove are mapped to move-

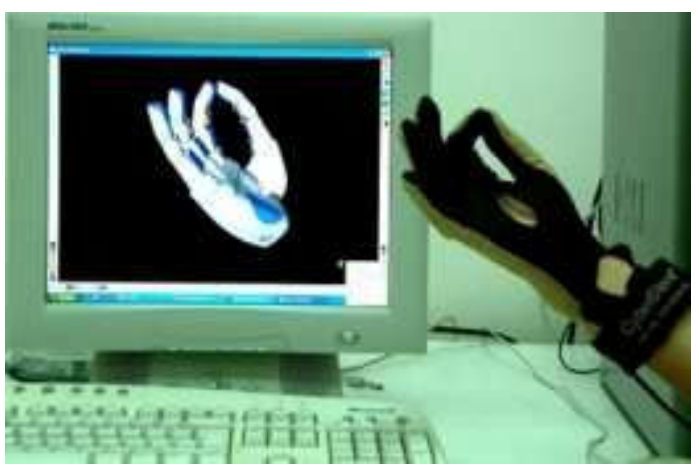

Fig. 2. Human hand with the sensorized glove connected to the mechanical hand simulator used in the data acquisition procedure.

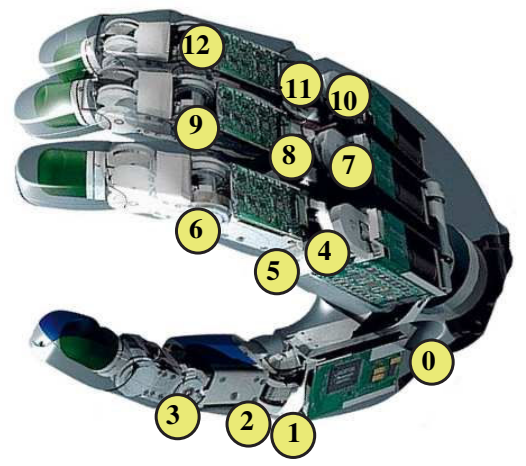

Fig. 3. The anthropomorphic mechanical hand used.

ments of the mechanical hand through a virtual simulator (Fig. 2), where the user has a visual feedback of the mapping (detailed in the next subsection). Thus, the user freely moves its hand in an unconstrained way, i.e. without performing any specific task, trying to cover the whole mechanical hand workspace. Mapped postures are recorded for processing and analysis.

\section{B. Mapping from the Sensorized Glove to the Mechanical Hand}

The Schunk antropomorphic hand (SAH) [20], shown in Fig. 3, is a 13-DOF robotic hand based on the DLR hand [5]. It has four identical fingers and one is equipped with an additional joint to function as the opposing thumb. Each finger has four joints, although the distal joint is mechanically coupled to the middle joint, i.e. there are three DOF per finger.

Since the SAH mimics the human hand movements, mapping the data from the glove sensors to the movements of the SAH is done in an almost direct way. The following issues are considered for the mapping (see Figures 1 and 3):

- The palm of the mechanical hand is rigid and therefore the palm arc sensor $v$ and the wrist flexion and abduction sensors $b$ and $a$ are ignored.

- The mechanical hand lacks the little finger and therefore the sensors $u, t, s$ and $r$ are ignored.

- The distal phalanx sensors $i, m$, and $q$ are not used since the SAH hand has a coupling between the medium and distal phalanx of each finger. 
TABLE I

CORRESPONDENCE BETWEen THE CyBERGLOVE SENSORS (FIG. 1) AND THE JOINTS OF THE SAH HAND (FIG. 3).

\begin{tabular}{|c|l|c|l|}
\hline \multicolumn{2}{|c|}{ Cyberglove Sensor } & \multicolumn{2}{|c|}{ SA Hand Joint } \\
\hline Id. & Name & Id. & Name \\
\hline $\mathrm{c}$ & thumb roll & 0 & thumb base \\
$\mathrm{c}$ & thumb roll & 1 & finger base (thumb) \\
$\mathrm{e}$ & thumb inner & 2 & proximal phalanx (thumb) \\
$\mathrm{f}$ & thumb outer & 3 & medium phalanx (thumb) \\
$\mathrm{j}$ & index abduction & 4 & finger base (index) \\
$\mathrm{g}$ & index inner & 5 & proximal phalanx (index) \\
$\mathrm{h}$ & index middle & 6 & medium phalanx (index) \\
- & medium abduction & 7 & finger base (medium) \\
$\mathrm{k}$ & medium inner & 8 & proximal phalanx (medium) \\
$\mathrm{l}$ & medium medium & 9 & medium phalanx (medium) \\
$\mathrm{n}$ & ring abduction & 10 & finger base (ring) \\
$\mathrm{o}$ & ring inner & 11 & proximal phalanx (ring) \\
$\mathrm{p}$ & ring medium & 12 & medium phalanx (ring) \\
\hline
\end{tabular}
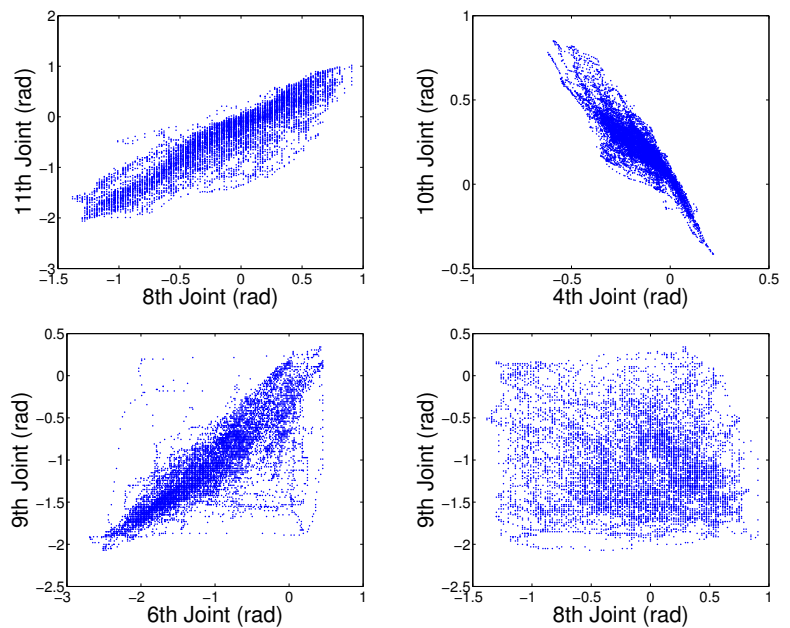

Fig. 4. Top-left: Positive correlation between proximal phalanxes (8 and 11); Top-right: Negative correlation between the abduction of the index and the ring (4 and 10); Bottom-left: Smooth positive correlation between medium phalanxes (6 and 9); Bottom-right: No correlation between consecutive phalanxes of the medium finger ( 8 and 9 ).

- Using the sensor $c$ to control joint 1 produces a more natural motion of the SAH hand than using sensor $d$, therefore sensor $c$ is used for both joints 0 and 1.

- In the glove, the abduction is measured in a relative way, i.e. sensors $j$ and $n$ give, respectively, the relative angle between the index and the middle fingers and between the middle and the ring fingers. Then, the mapping is done using the middle finger as reference, i.e. the base of the middle finger (joint 7) is fixed to zero, and sensors $j$ and $n$ are directly associated to joints 4 and 10 , respectively.

Then, only 11 values from the 22 available in the glove are used in the mapping to the joints of the SAH mechanical hand. The complete mapping is shown in Table I. Note that this mapping makes the motions of the $\mathrm{SAH}$ hand to be defined with 11 independent parameters, although it has 13 DOF.

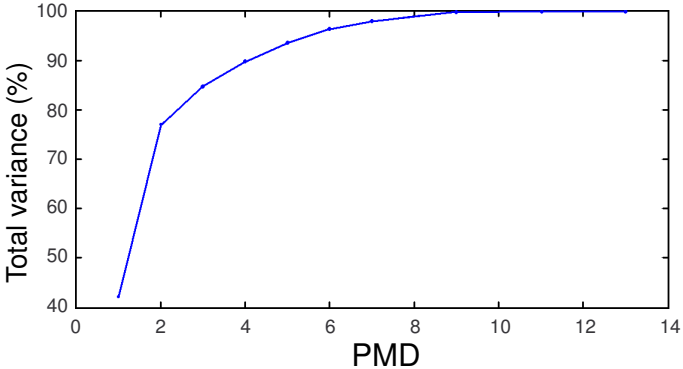

Fig. 5. Total variance covered when using an increasing number of PMDs

\section{Principal Motion Directions}

Dimensionality reduction of a feature set is a common preprocessing step used for pattern recognition and classification applications as well as in compression schemes. Principal component analysis (PCA) is often used in these fields to reduce multidimensional data sets to lower dimensions for analysis [21]. It is also used as a tool in exploratory data analysis and for making predictive models. PCA involves the computation of the eigenvalue decomposition of a data covariance matrix or the singular value decomposition of a data matrix, usually after mean centering the data for each attribute.

In this work, PCA is used to reduce the configuration space of the mechanical hand SAH to a more tractable space of smaller dimension, using the data recorded from the hand postures (Section III-A). Fig. 4 shows some examples of the existing correlation between joints, illustrating that an effective reduction can be obtained.

The vectors that define the new base of the hand space are called Principal Motion Directions (PMDs). Selecting only the first vectors with higher associated variances a reduced hand space is obtained: The first PMD represents the $42.19 \%$ of the total variance in the analyzed dataset; the first two components the $77.12 \%$, and the first three components the $84.71 \%$ (the complete evolution is shown in Fig. 5). Therefore, in this work the use of three PMDs has been considered sufficient. Fig. 6 shows the hand postures along the two principal components, and Fig. 7 the postures resulting from their linear combination.

\section{MOTION PLANNING}

Sampling-based motion planners have demonstrated to be one of the best alternatives for path planning problems, since they avoid the explicit characterization of the obstacles of the configuration space $\mathcal{C}$. These planners generate collisionfree samples of $\mathcal{C}$ and connect them with free paths capturing the connectivity of the free space either by forming roadmaps [22] or trees [23].

A sampling-based roadmap planner, using a deterministic sampling sequence as sampling source, will be used here to find the motions of the hand and of the arm where it is mounted. It relies on generating samples from the lower dimensional space $\mathcal{S C}$ obtained by considering, for the hand motions, the subspace $\mathcal{S} \mathcal{C}_{\mathrm{h}}$ defined by the first three PMDs. 


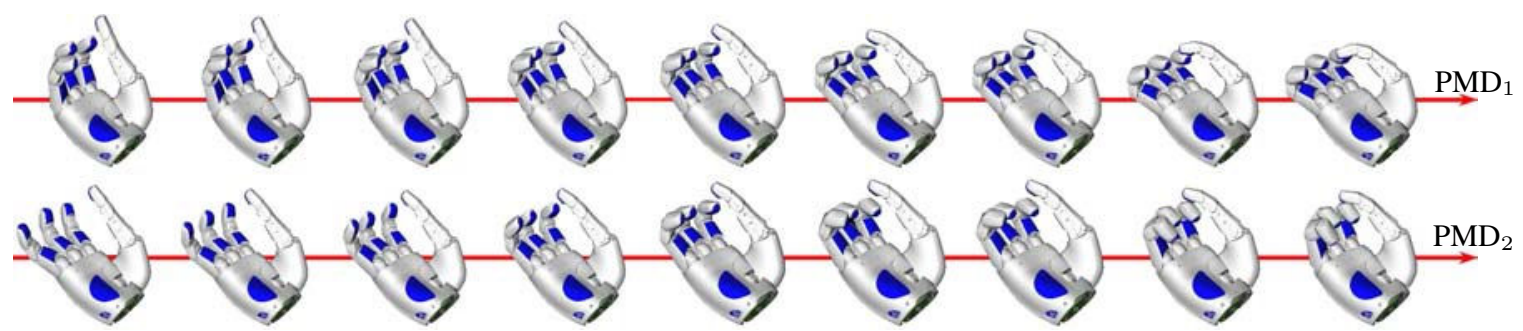

Fig. 6. Configurations of the SAH hand when it is moved along the first two PMDs.

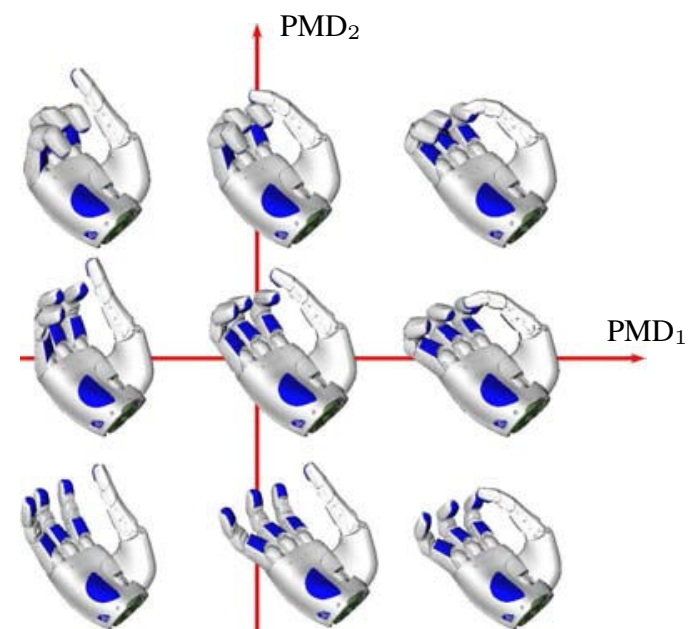

Fig. 7. Configurations of the SAH hand when it is moved along a combination of the first two PMDs.

\section{A. The algorithm}

A basic sampling-based roadmap planner has an initial preprocessing phase to construct the graph that represents the roadmap capturing the connectivity of the free configuration space $\left(\mathcal{C}_{\text {free }}\right)$. The nodes of the graph are the configurations sampled from $\mathcal{C}_{\text {free }}$ and the edges the collision-free paths that connect them.

Fig. 8 shows the algorithm that returns the graph $G$ representing the roadmap. The input is the number $N$ of configurations to sample. The functions used are:

- Function INSERT $(s, V)$ : inserts a node to the set $V$ of nodes of the graph. The use of an efficient graph structure like the ones provided by the Boost Graph Library [24] greatly enhances the implementation.

- Function InSERT $((s, q), E)$ : inserts the edge $(s, q)$ connecting configurations $s$ and $q$ to the set $E$ of edges of the graph.

- Function get-SAmple(): generates a configuration of $\mathcal{S C}$, using the PMDs for the hand configurations, as it is detailed in the following subsection.

- Function fREE $(s)$ : returns tRUe if the configuration $s$ belongs to $\mathcal{C}_{\text {free }}$. The use of a simple and efficient collision detection library like $P Q P[25]$ is mandatory since this is a time-consuming step of the process.

- Function NEIGBORHOOD(s): returns the set of up to $K$ samples that lie within a predefined neighborhood of $s$. This can be efficiently implemented using either the

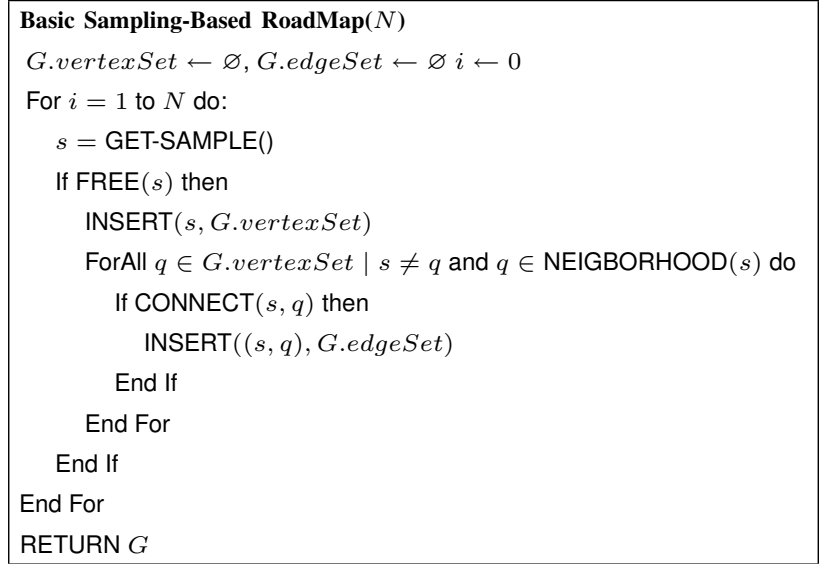

Fig. 8. Algorithm for the preprocessing phase of a basic sampling-based roadmap planner.

MPNN algorithm [26] or taking advantage of the grid structure if grid-based deterministic sampling sequences are used [27].

- Function $\operatorname{CONNECT}(s, q)$ : determines whether the rectilinear path in $\mathcal{C}$ connecting $s$ and $q$ is free or not by performing the collision-check test to several of its configurations. This can be efficiently done using the binary method [28].

The second phase of a sampling-based roadmap planner is the query phase where the initial and the goal configurations $\left(C_{o}\right.$ and $\left.C_{d}\right)$ are connected to the roadmap, and graph search algorithms are used to find a path connecting them. In the proposed implementation, the initial and the goal configurations of the hand are free configurations that are not constrained to lie in the subspace $\mathcal{S C}_{\mathrm{h}}$ defined by the PMDs, but they can be any configuration of $\mathcal{C}_{\mathrm{h}}$. The connection of $C_{o}$ and $C_{d}$ to the roadmap is done searching for free paths in $\mathcal{C}$ between these configurations and the nearest nodes in the roadmap, using the $\operatorname{CONNECT}(s, q)$ function. The search algorithm used is the $A^{*}$.

\section{B. The sampling source}

Sampling-based methods usually rely on the use of a random number generation source, although the use of deterministic sampling sequences is a good alternative [29]. Deterministic sampling sequences provide an incremental and uniform coverage of $\mathcal{C}$, with a better dispersion than random sampling. Deterministic sampling has given slightly better results than random sampling in roadmap planners [30] 


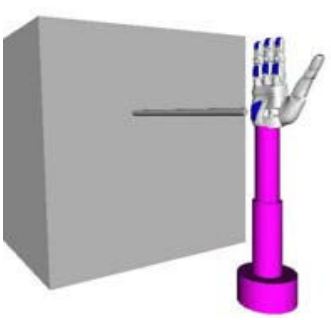

Fig. 9 .

Fig. 9. Configurations: a) $C_{o}$ and b) $C_{d}$.

(although Hsu et al. [31] constrained this improvement to few degrees of freedom tasks and considered it much less significant than the importance sampling issue devoted to bias the samples towards regions relevant to the task). With the objective of sampling uniformly over the space defined by the first main PMDs, the use of a deterministic sampling sequence is therefore a good option. In this work, the $s_{d}(k)$ deterministic sequence has been chosen [32]. It is a deterministic sequence based on a multi-grid cell decomposition and on the use of the digital construction method first proposed in [33]. The $s_{d}(k)$ sequence is a sequence of cells of the maximum partition level. Samples are random configurations within those cells, with coordinates in the range $[0,1]$.

Let $H=\operatorname{dim}\left(\mathcal{C}_{\mathrm{h}}\right), A=\operatorname{dim}\left(\mathcal{C}_{\mathrm{a}}\right)$, and $h=\operatorname{dim}\left(\mathcal{S} \mathcal{C}_{\mathrm{h}}\right)$. The function GET-SAMPLE() uses the $s_{d}(k)$ sequence to obtain a $d$-dimensional sample from $\mathcal{S C}$, with $d=A+h$. The first $A$ components are scaled to obtain the $A$ joint coordinates of the arm within the corresponding joint ranges; the last $h$ components are used to obtain the $H$ joint coordinates of the mechanical hand as follows. Let:

- $\mathbf{e}_{i}, i=1, \ldots, h$, be the unitary vectors defining the first $h$ PMDs (when ordered in a decreasing order of the variance given by the corresponding eigenvalues $\lambda_{i}$, of the eigenvalue decomposition of the data covariance matrix).

- $\mathbf{b}=\left(b_{1}, \ldots, b_{H}\right)^{T}$ be the data mean (Section III-C).

- $\Delta_{i}=4 \sqrt{\lambda_{i}}$ be the range (centered at b) covered by the 95\% of the dataset along the direction defined by $\mathbf{e}_{i}$.

- $\mathbf{E}$ be a $H \times h$ matrix defined as $\mathbf{E}=\left[\Delta_{1} \mathbf{e}_{1}, \ldots, \Delta_{h} \mathbf{e}_{h}\right]$.

- $\mathbf{p}=\left(p_{1}, \ldots, p_{h}\right)^{T}$ be the last $h$ components of the sample generated by the $s_{d}(k)$ sequence, shifted by -0.5 along each component, i.e. $-0.5 \leq p_{i} \leq 0.5$ (recall that $s_{d}(k)$ gives values within $[0,1]$ and sample p should span the joint coordinates around $\mathbf{b}$ ).

Then, the joint values $\boldsymbol{\Theta}=\left(\theta_{1}, \ldots, \theta_{H}\right)^{T}$ of the mechanical hand are obtained as follows:

$$
\Theta=\mathbf{E} \mathbf{p}+\mathbf{b}
$$

\section{EVALUATION AND DISCUSSION}

The evaluation of the proposed approach is done by comparing its efficiency with that of a basic probabilistic roadmap planner. Fig. 9 shows the initial and goal configurations $\left(C_{o}\right.$ and $\left.C_{d}\right)$ of the planning problem to be solved, where the $\mathrm{SAH}$ hand is mounted on a robot with three revolute joints. Therefore, considering three PMDs, $\mathcal{S C}$ is
TABLE II

COMPARISON BETWEEN APPROACHES FOR THE TEST CASE

\begin{tabular}{|c|c|c|c|}
\hline & \# samples & \multicolumn{2}{|c|}{ \# nodes } \\
\cline { 3 - 4 } & Mean & Mean & $95 \%$ Confidence Interval \\
\hline 16-RND & 63 & 21 & {$[12.9,28.3]$} \\
\hline 6-SDK & 25 & 15 & {$[12.0,17.9]$} \\
\hline
\end{tabular}

6-dimensional (this scenario is called 6-SDK). On the other hand, the basic PRM samples randomly over all the joint space, and therefore the space $\mathcal{C}$ where the planning is done is 16-dimensional (this scenario is called 16-RND).

Table II shows the summary results of 10 trials per scenario. It can be seen that the proposed approach is able to solve the problem with less samples than the basic PRM, and with less variability between trials. One reason for this efficiency is that the sampling over PMDs provides (self)collision-free samples more often than sampling over all the joint space, and with hand-postures that mimic those of the human hand, being therefore best suited to avoid collisions with the objects to be grasped.

Aside from the quantitative results, it is worth noting that the motions obtained with the proposed approach look more natural from the anthropomorphic and aesthetic points of view, as it can be appreciated in Fig. 10 where two sequences of snapshots are shown, respectively, for the 16-RND and 6-SDK case. This advantage is less evident if a postprocessing smoothing is done in the 16-RND case, although this adds an extra computational cost.

\section{CONCLUSIONS}

This paper has presented an efficient methodology to compute collision-free motions of a hand-arm system based on the principal motions direction (PMDs). These directions, obtained by demonstration using pattern recognition techniques, capture the natural motion of the human hand. Taking the PMDs with more weight (those with larger variances), allows a reduction of the dimension of the hand-movement space that greatly eases the work of a sampling-based roadmap planner. Added to a good computational efficiency, the hand motions obtained by the proposed planner are more natural than those obtained when all the degrees of freedom of the hand are directly sampled using a random sampling sequence in the joint space. Currently, the proposed planner is being enhanced by the use of an importance sampling method to bias the samples towards more relevant regions of the configuration space, in order to perform tasks with smaller clearances. Future work includes the implementation of rapidly-exploring random trees to quickly solve single queries.

\section{REFERENCES}

[1] E. Peña, J. Yang, and K. Abdel-Malek, "Santos ${ }^{\mathrm{TM}}$ hand: A 25-degreeof-freedom model," in Proc. of SAE Digital Human Modeling for Design and Engineering, 2005.

[2] S. C. Jacobsen, J. E. Wood, D. F. Knutti, and K. B. Biggers, "The UTAH/M.I.T. dextrous hand: Work in progress," The Int. J. of Robotics Research, vol. 3, no. 4, pp. 21-50, December 1984. 

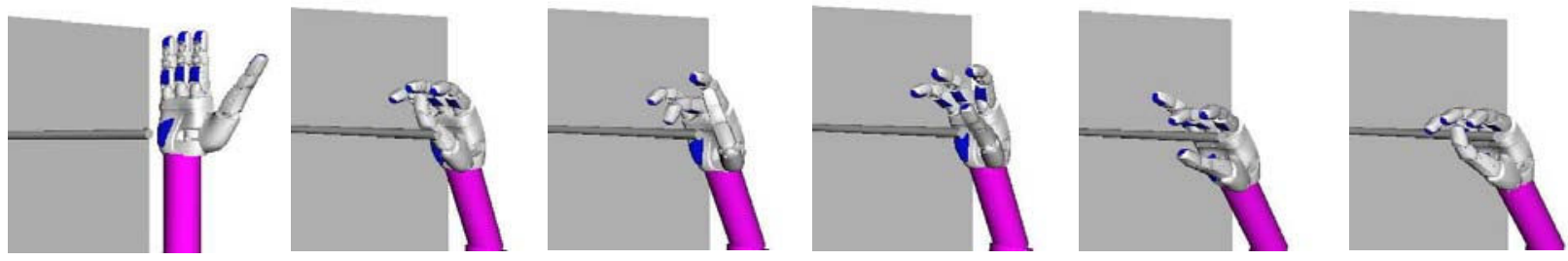

a) Solution found with the 16-RND approach
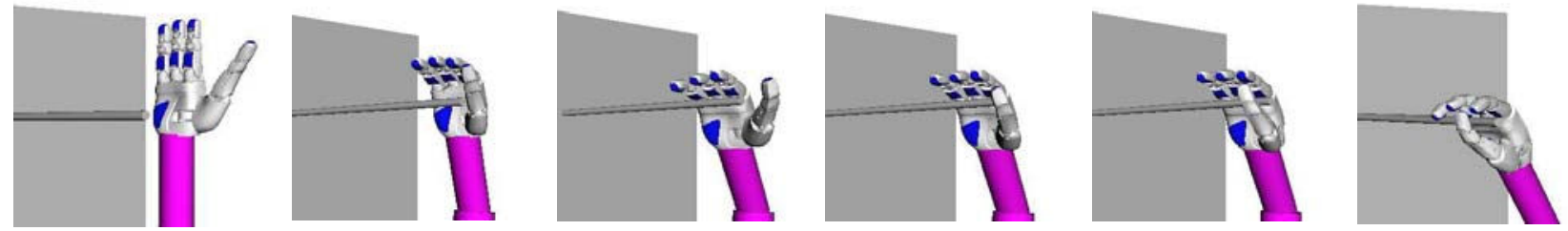

b) Solution found with the 6-SDK approach

Fig. 10. Snapshots of one of the successful attempts to connect the given $C_{o}$ and $C_{d}$ configurations (see the accompanying video for the whole sequence).

[3] A. Caffaz and G. Cannata, "The design and development of the DISTHand dextrous gripper," in Proc. of the IEEE Int. Conf. on Robotics and Automation, vol. 3, 1998, pp. 2075-2080.

[4] J. P. Gazeau, S. Zehloul, M. Arsicault, and J. P. Lallemand, "The LMS hand: force and position controls in the aim of the fine manipulation of objects," in Proc. of the IEEE Int. Conf. on Robotics and Automation, vol. 3, 2001, pp. 2642-2648.

[5] J. Butterfass, M. Fischer, M. Grebenstein, S. Haidacher, and G. Hirzinger, "Design and experiences with DLR hand II," in Proc. of the World Automation Congress, vol. 15, 2004, pp. 105-110.

[6] R. Suárez and P. Grosch, "Mechanical hand MA-I as experimental system for grasping and manipulation," in Video Proc. of the IEEE Int. Conf. on Robotics and Automation, Barcelona, 2005.

[7] G. A. Bekey, R. Tomovic, and I. Zeljkovic, Control architecture for the Belgrade/USC hand. Springer-Verlag New York, Inc., 1990, pp. 136-149.

[8] M. S. Ali, K. J. Kyriakopoulos, and H. E. Stephanou, "The kinematics of the anthrobot-2 dextrous hand," in Proc. of the IEEE Int. Conf. on Robotics and Automation, 1993, pp. 705-710 vol.3.

[9] L.-R. Lin and H.-P. Huang, "Mechanism design of a new multifingered robot hand," in Proc. of the IEEE Int. Conf. on Robotics and Automation, vol. 2, 1996, pp. 1471-1476.

[10] C. S. Lovchik and M. A. Diftler, "The robonaut hand: a dexterous robot hand for space," in Proc. of the IEEE Int. Conf. on Robotics and Automation, vol. 2, 1999, pp. 907-912.

[11] Shadow Robot Company, "Design of a dextrous hand for advanced clawar applications," in Proc. of the Climbing and Walking Robots and the Supporting Technologies for Mobile Machines: CLAWAR, 2003, pp. 691-698.

[12] H. Kawasaki, T. Komatsu, and K. Uchiyama, "Dexterous anthropomorphic robot hand with distributed tactile sensor: Gifu hand II," IEEE/ASME Trans. on Mechatronics, vol. 7, no. 3, pp. 296-303, 2002.

[13] F. Lotti, P. Tiezzi, G. Vassura, L. Biagiotti, G. Palli, and C. Melchiorri, "Development of UB hand 3: Early results," in Proc. of the IEEE Int. Conf. on Robotics and Automation, 2005, pp. 4488-4493.

[14] A. Bicchi, "Hands for dexterous manipulation and robust grasping: a difficult road toward simplicity," IEEE Trans. on Robotics and Automation, vol. 16, no. 6, pp. 652-662, 2000.

[15] L. Biagiotti, F. Lotti, C. Melchiorri, and G. Vassura, "How far is the human hand? a review on anthropomorphic robotic end-effectors," University of Bologna, Tech. Rep., 2004.

[16] M. F. M. Santello and J. F. Soechting, "Postural hand synergies for tool use," The Journal of Neuroscience, vol. 18, no. 23, pp. 10 105-10 115, December 1998.

[17] M. Ciocarlie, C. Goldfeder, and P. Allen, "Dimensionality reduction for hand-independent dexterous robotic grasping," in Proc. of the IEEE/RSJ Conf. on Intelligent Robots and Systems, 2007, pp. 32703275 .
[18] A. Tsoli and O. C. Jenkins, "2d subspaces for user-driven robot grasping," in Proc. of the RSS 2007 Workshop on Robot Manipulation: Sensing and Adapting to the Real World, 2007.

[19] A. Safonova, J. K. Hodgins, and N. S. Pollard, "Synthesizing physically realistic human motion in low-dimensional, behavior-specific spaces," ACM Trans. Graph., vol. 23, no. 3, pp. 514-521, 2004.

[20] Schunk GmbH \& Co. KG, "Schunk anthropomorphic hand," http://www.schunk.com/, 2006.

[21] J. I.T., Principal Component Analysis. Upper Saddle River, NJ, USA: Springer Series in Statistics, 2002.

[22] L. E. Kavraki and J.-C. Latombe, "Randomized preprocessing of configuration for fast path planning," in Proc. of the IEEE Int. Conf. on Robotics and Automation, vol. 3, 1994, pp. 2138-2145.

[23] J. J. Kuffner and S. M. LaValle, "RRT-connect: An efficient approach to single-query path planning," in Proc. of the IEEE Int. Conf. on Robotics and Automation, 2000, pp. 995-1001.

[24] L.-Q. Lee, J. G. Siek, and A. Lumsdaine, "The generic graph component library," in 14th ACM SIGPLAN Conf. on Object-oriented Programming, Systems, Languages and Applications., 1999, pp. 399414.

[25] M. C. L. Eric Larsen, Stefan Gottschalk and D. Manocha, "Fast proximity queries with swept sphere volumes," in Proc. of Int. Conf. on Robotics and Automation, 2000, pp. 3719-3726.

[26] A. Yershova and S. LaValle, "Improving motion planning algorithms by efficient nearest-neighbor searching," IEEE Trans. on Robotics, vol 23(1), pp. 151 - 157, 2006.

[27] J. Rosell and A. Pérez, "Sampling C-obstacles border using a filtered deterministic sequence," Technical report IOC-UPC-DT-P-11-2008, Tech. Rep., September 2008.

[28] R. Geraerts and M. H. Overmars, "Sampling and node adding in probabilistic roadmap planners," Robotics and Autonomous Systems, vol. 54, p. 165173, 2006

[29] M. S. Branicky, S. M. LaValle, K. Olson, and L. Yang, "Quasirandomized path planning," in Proc. of the IEEE Int. Conf. on Robotics and Automation, 2001, pp. 1481-1487.

[30] S. M. LaValle, M. S. Branicky, and S. R. Lindemann, "On the relationship between classical grid search and probabilistic roadmaps," Int. J. of Robotics Research, vol. 23, no. 7-8, pp. 673-692, 2004.

[31] D. Hsu, J.-C. Latombe, and H. Kurniawati, "On the probabilistic foundations of probabilistic roadmap planning," Int. Journal of Robotics Research, vol. 25, no. 7, pp. $627-643,2006$

[32] J. Rosell, M. Roa, A. Pérez, and F. García, "A general deterministic sequence for sampling d-dimensional configuration spaces," $J$. of Intelligent and Robotic Systems, vol. 50, no. 4, pp. 361-374, 2007.

[33] S. R. Lindemann, A. Yershova, and S. M. LaValle, "Incremental grid sampling strategies in robotics," in Proc. of the Sixth Int. Workshop on the Algorithmic Foundations of Robotics, 2004, pp. 297 - 312. 